\title{
New functionalised polymeric microspheres for multicomponent solid phase extraction of phenolic compounds
}

\author{
Magdalena Sobiesiak $^{1} \cdot$ Beata Podkościelna $^{1} \cdot$ Przemysław Podkościelny $^{2}$
}

Received: 11 October 2015/Revised: 30 November 2015/Accepted: 22 December 2015/Published online: 4 January 2016

(C) The Author(s) 2015. This article is published with open access at Springerlink.com

\begin{abstract}
Three new porous copolymers possessing basic character were prepared by chemical modification of polymeric adsorbent (copolymer of bis[4(2-hydroxy-3methacryloyloxypropoxy)phenyl]sulfide and glycidyl methacrylate, BES.DM + GMA). Modification was carried out by reactions of surface epoxide groups of the precursor with amines: butylenediamine, diethylenetriamine and triethylenetetramine. Next, two of the synthesized materials were chosen for further modification involving addition of $\mathrm{HCO}_{3}{ }^{-}$groups to amine species. The obtained polymer adsorbents were characterised by elemental analyses, FTIR, nitrogen adsorption and thermal analyses. The prepared copolymers were studied for use as adsorbent in solid phase extraction (SPE) of multicomponent mixture of phenolic compounds consisting of: phenol, 2-chlorophenol, 2,4-dichlorophenol and 2,4,6trichlorophenol. The SPE experiments were carried out in off-line mode. The influence of the chemical modification on adsorption properties of the presented materials was observed. The prepared adsorbents possessed different sorption properties towards the phenols. In addition, a competition phenomenon of the testing compounds for active sites on the polymer surface was observed. The most effective in adsorption of phenol and its chlorinated derivatives were the polymers with both amine and
\end{abstract}

Beata Podkościelna

beatapod@poczta.umcs.lublin.pl

1 Department of Polymer Chemistry, Faculty of Chemistry, Maria Curie-Skłodowska University, Pl. M. C. Skłodowskiej 5, 20-031 Lublin, Poland

2 Department of Theoretical Chemistry, Faculty of Chemistry, Maria Curie-Skłodowska University, Pl. M. C. Skłodowskiej 5, 20-031 Lublin, Poland bicarbonate groups. The best recovery results were obtained for 2,4-dichlorophenol.

Keywords SPE - Phenolic compounds - Modification of polymers $\cdot$ Polymeric sorbents $\cdot$ Water purification

\section{Introduction}

Nowadays, polymers are widely used for various applications. Their chemical resistance makes them materials difficult to replace. Moreover, their structure and chemical character can be modified in many ways and adjusted for specific purposes (Fontanals et al. 2007; Pan et al. 2005). That is why polymers are invariably under researchers' investigation.

The search of new polymeric materials, used as sorbents, is particularly important from the point of view of environmental protection. Many chemical pollutants possess polar character. In order to isolate such substances from environmental matrices, polar sorbents can be used. Chemical modification of already existing polymeric sorbents or designing new ones can help to solve the problem of removing from the environment a large variety of polar pollutants (Feng et al. 2009; Rodil et al. 2012).

The ability to interact with living organisms and the inanimate matter of nature, as well as the chemical properties of compounds strongly depend on their structure. The kind, number and position of substituents may alter substantially the way of interaction of a molecule with its surroundings ( $\mathrm{Lu}$ and Sorial 2007). Phenol and its chlorinated derivatives belong to the same group of phenolic compounds, however their properties and toxicity vary in a wide range. The enhanced attraction with the surface in the case of chlorophenols is due to the electron-withdrawal 
phenomenon of the chlorine substituents, as they reduce the overall electron density of the aromatic ring (Podkoscielny and Nieszporek 2011; László et al. 2003). Phenol is more quickly biodegradable, whereas chlorophenols are biodegradable relatively slow (Olaniran and Igbinosa 2011). Phenols are very toxic even at low concentrations and cause an unpleasant odour and taste of water.

The value of logarithm of the octanol-water partition coefficient $\left(\mathrm{K}_{\mathrm{ow}}\right)$, i.e. $\log \mathrm{K}_{\mathrm{ow}}$ is often used as basic bioaccumulation criterion. If its value is in the range of $1-3$, bioaccumulation is estimated as low or moderate, for values higher than 3 , substances are considered as possessing high accumulation potential. For comparison of toxic properties of the phenols used in this study, two parameters were chosen- $\mathrm{LD}_{50}$ for rat and $\mathrm{EC}_{50}$ for Daphnia magna.

According to the data presented in Table 1 (GESTIS and PubChem databases), the most toxic substance among those mentioned is 2,4-dichlorophenol but all the quoted compounds are poisoning after dissolving in water. As a consequence, the European Union classified phenols as priority contaminants and the 80/778/EC directive stated a maximum concentration of $0.5 \mu \mathrm{g} / \mathrm{L}$ for total phenols in drinking water, and individual concentration cannot exceed $0.1 \mu \mathrm{g} / \mathrm{L}$ (Drinking Water Directive 80/778/EEC).

Phenolic compounds are introduced to environmental water as a consequence of industrial, agricultural, and communal activities of humans. Their quantitative isolation from aqueous environment is often very difficult because of their polarity and low concentrations ( $\mathrm{Lu}$ and Sorial 2007; Simpson 2000; Zhong et al. 2012; Lu and Sorial 2004). For this reason a preconcentration step is often included in analytical process. Nowadays, a very popular method of isolation and preconcentration is solid phase extraction (SPE). In order to make it more efficient, new types of packaging are still researched (Feng et al. 2009; Rodríguez et al. 2000; Sobiesiak and Podkoscielna 2010; Sobiesiak 2011; Castillejos et al. 2011; An et al. 2012; Sobiesiak 2012; Puziy et al. 2013).
This work presents studies of four newly prepared polymer-based adsorbents in the form of microspheres, used in the off-line SPE method for preconcentration of phenolic compounds. The synthesised materials possess functional groups with polar character that is important for phenols sorption process. The parent copolymer microspheres were prepared by suspension-emulsion polymerization of two monomers, bis[4(2-hydroxy-3methacryloyloxy-propoxy)phenyl]sulfide (BES.DM) and glycidyl methacrylate (GMA) in presence of pore forming diluents (toluene/decan-1-ol). The obtained parent microspheres possessed in their structure reactive epoxide groups which were modified with three amines: butylenediamine (BDA), diethylenetriamine (DETA) and triethylenetetramine (TETA). Next, two of the obtained materials, BES.DM + GMA + BDA and BES.DM + GMA + DETA were additionally modified in order to introduce $\mathrm{HCO}_{3}{ }^{-}$groups.

Depending on the way of modification, the prepared sorbents possessed different sorption properties toward the phenols used in the SPE experiment.

\section{Experimental}

\subsection{Chemicals and eluents}

Glycidyl methacrylate (GMA), decan-1-ol, bis(2-ethylhexyl)sulfosuccinate sodium salt (DAC, BP), butylenediamine (BDA), diethylenetriamine (DETA) and triethylenetetramine (TETA) were from Fluka AG (Buchs, Switzerland). $\quad \alpha, \alpha^{\prime}$-Azoiso-bis-butyronitrile (AIBN), methanol $(\mathrm{MeOH})$ for chromatography, 2-chlorophenol (ChP), 2,4-dichlorophenol (DChP) and 2,4,6-trichlorophenol (TChP) were purchased from Merck (Darmstadt, Germany). Reagent grade $\mathrm{NaHCO}_{3}, \mathrm{HCl}$, hexane, toluene and analytical grade phenol $(\mathrm{PH})$ were bought from $\mathrm{POCh}$ (Gliwice, Poland).

Table 1 Chemical properties and toxicity of phenol and its chlorinated derivatives

\begin{tabular}{lllll}
\hline & Phenol & 2-chlorophenol & 2,4-dichlorophenol & 2,4,6-trichlorophenol \\
\hline State of aggregation & Solid & Liquid & Solid & Solid \\
Density $\left(\mathrm{g} / \mathrm{cm}^{3}\right)$ & 1.06 & 1.26 & 1.38 & 1.68 \\
Partition coefficient (octanol/water) $\log \mathrm{K}_{\mathrm{ow}}$ & 1.46 & 2.15 & 3.06 & 3.69 \\
Biodegrability $(\% /$ days) & $85 / 14$ & $0 / 14$ & $0 / 28$ & 47 \\
LD $_{50}$ oral, rat $(\mathrm{mg} / \mathrm{kg})$ & 317 & 670 & 1.4 & 3.3 \\
Toxicity for Daphnia magna $\mathrm{EC}_{50}(\mathrm{mg} / \mathrm{L}, 48 \mathrm{~h})$ & 4.2 & 7.4 & \\
\hline
\end{tabular}

$L D_{50}$ median lethal dose (dose required to kill half the population used in a test) generally used as indicator of acute toxicity of chemicals for animals

$E C_{50}$ effective concentration of a tested compound causing response of $50 \%$ population after a specified time of exposition. This parameter is used as a risk factor for natural life existing in aquatic environment 
Bis[4(2-hydroxy-3-methacryloyloxypropoxy)phenyl]sulfide (BES.DM) was obtained by the procedure described elsewhere (Podkościelna et al. 2009).

\subsection{Synthesis of polymeric microspheres (BES.DM + GMA)}

The synthesis was carried out in a three-necked flask equipped with a stirrer, a water condenser and a thermometer. First, the dispersion medium was prepared through the dissolution of $1 \mathrm{wt} \%$ bis(2-ethylhexyl)sulfosuccinate sodium salt (DAC, BP) in deionised water $\left(150 \mathrm{~cm}^{3}\right)$. Next, the mixture of monomers: BES.DM $(10 \mathrm{~g})$ and GMA $(2.84 \mathrm{~g})$ were made and the initiator AIBN $(1.0 \mathrm{wt} \%)$ was added. Then, the prepared solution was diluted with a mixed solvent (toluene/decan-1-ol) and poured to the dispersion medium. The reaction mixture was stirred at $350 \mathrm{rpm}$ for $18 \mathrm{~h}$ at $80{ }^{\circ} \mathrm{C}$ (Podkościelna 2011). The obtained copolymers were washed with distilled water, filtered off, dried and extracted (acetone and methanol) in a Soxhlet apparatus.

\subsection{Modifications of (BES.DM + GMA) copolymer}

The copolymers were modified with amines in the epoxide ring opening reaction. In a $250 \mathrm{~cm}^{3}$ round bottomed twonecked flask equipped with a mechanical stirrer and a thermometer, selected microspheres were placed together with BDA or DETA or TETA and toluene (Podkościelna 2013). The whole content was heated over a water bath at $80^{\circ} \mathrm{C}$ for $6 \mathrm{~h}$ and next kept for $18 \mathrm{~h}$ at room temperature. The obtained modified microspheres were washed with distilled water, filtered off, dried and extracted in a Soxhlet apparatus with boiling toluene. Next, BES.DM + G$\mathrm{MA}+\mathrm{BDA}$ and BES.DM + GMA + DETA derivatives were reacted with $\mathrm{H}_{2} \mathrm{CO}_{3}\left(\mathrm{NaCO}_{3}+\mathrm{H}_{2} \mathrm{SO}_{4}\right)$ in order to modify the primary amino groups by addition of $\mathrm{HCO}_{3}{ }^{-}$ groups. The reaction was carried out at room temperature for about $2 \mathrm{~h}$.

\subsection{Characterization of obtained sorbents}

The porous structure of the studied sorbents was characterized by nitrogen adsorption-desorption isotherms measured at $77 \mathrm{~K}$ using adsorption analyser ASAP 2405 (Micromeritics Inc., USA). Before analyses, the samples were outgassed at $140{ }^{\circ} \mathrm{C}$ for $1 \mathrm{~h}$.

ATR-FTIR spectra were obtained using a Bruker FTIR spectrophotometer TENSOR 27.

Elemental analyses were made on a Perkin Elmer CHN 2400 analyser (Palo Alto, CA, USA).

Thermogravimetric analyses (TG/DTG) were performed with a thermal analyzer STA 449 F1 Jupiter (Netzsch, Selb, Germany). $\mathrm{Al}_{2} \mathrm{O}_{3}$ pans were used for measurements. The sample weight was about $7 \mathrm{mg}$. Dynamic scans were performed at the heating rate of $10 \mathrm{~K} / \mathrm{min}$ in the temperature range $30-1000{ }^{\circ} \mathrm{C}$, under helium atmosphere $(40 \mathrm{~mL} / \mathrm{min})$.

Surface chemistry of the materials was characterised by $\mathrm{pH}$ of the slurry and modified Boehm's titration method (Boehm 1994). Weighed amounts of sorbents $(200 \pm 1 \mathrm{mg})$ were placed into an Erlenmeyer flask and $20 \mathrm{~mL}$ of either $0.05 \mathrm{M} \mathrm{NaOH}$ (for Cation Exchange Capacity measurement) or $0.05 \mathrm{M} \mathrm{HCl}$ (for Anion Exchange Capacity measurement) solution was added. Samples were equilibrated for $24 \mathrm{~h}$. Next, the concentration of base or acid was measured by titration with $\mathrm{HCl}$ or $\mathrm{NaOH}$, respectively. Calculated values of CEC or AEC were expressed in $\mathrm{mmol} / \mathrm{g}$.

\subsection{Solid phase extraction (SPE) experiments}

SPE method was applied to estimate sorption abilities of the prepared porous polymers towards phenol and its chlorinated derivatives. Phenols were preconcentrated from aqueous solution using laboratory cartridges filled with $100 \mathrm{mg}$ of the studied material. The aqueous solutions of the phenolic compounds were prepared by dilution (1:50 $\mathrm{v} / \mathrm{v}$ ) of a standard methanolic solution containing $100 \mathrm{mg} /$ $\mathrm{L}$ of phenol (PH), 2-chlorophenol (ChP), 2,4-dichlorophenol (DChP), and 2,4,6-trichlorophenol (TChP). Initial concentration of phenols in aqueous solution was $2 \mathrm{mg} / \mathrm{L}$. Different volumes of the water solutions were sucked through the cartridge immersed in the sample and connected by PTFE tubing (Chrompack, Middelburg, Netherlands) to the water aspirator. After the sample had passed through the cartridge, the vacuum was maintained for 5 min to dry the sorbent bed. Next, the phenolic compounds were eluted from cartridge with adequate volume of methanol $(2 \mathrm{~mL}$ of $\mathrm{MeOH}$ for each $100 \mathrm{~mL}$ of sucked water solution). In order to determine the recoveries of the eluted phenols, the obtained eluates were analysed using HPLC system. Details of the analysis were described elsewhere (Sobiesiak and Podkoscielna 2010; Sobiesiak 2011; Gawdzik et al. 2005). Recovery values were calculated as the mean of three determinations. Decrease of the recovery below $25 \%$ of maximum value was assumed as a breakthrough volume for the compound considered.

\section{Results and discussion}

\subsection{Structure and characteristic of polymers}

Chemical structures of the studied monomers and the schemes of their copolymerisation and modification are presented in Fig. 1. Additionally, the polymeric 
microspheres are shown in Fig. 2. The image was taken using optical microscope Motic (China).

\subsubsection{Characterization of porous structure}

Characteristic parameters of porous structure of the polymers studied are presented in Table 2. Specific surface areas $\left(\mathrm{S}_{\mathrm{BET}}\right)$ and total pore volumes $\left(\mathrm{V}_{\mathrm{tot}}\right)$ are in the range of $27-55 \mathrm{~m}^{2} / \mathrm{g}$ and $0.2-0.4 \mathrm{~cm}^{3} / \mathrm{g}$, respectively. These materials are mesoporous. Average pore sizes (W) are larger than $20 \mathrm{~nm}$ (Fig. 3). Additionally, the percentage share of micropore volumes $\left(\mathrm{V}_{\mathrm{mi}}\right)$ in porous structure of the polymers is below $1 \%$. According to the values of the porous structure parameters presented in Table 2 and in Fig. 3, the topographic structure of the studied materials is similar.

Higher values of porous structure parameters are observed for the polymers modified with $\mathrm{HCO}_{3-}$. This means that bicarbonate ions acted not only as a reagent modifying chemical structure, but also promoted the development of polymer porosity.

\subsubsection{ATR/FTIR spectra}

In Fig. 4, ATR-FTIR spectra of BES.DM + GMA and its modified derivatives are shown. All the spectra are very

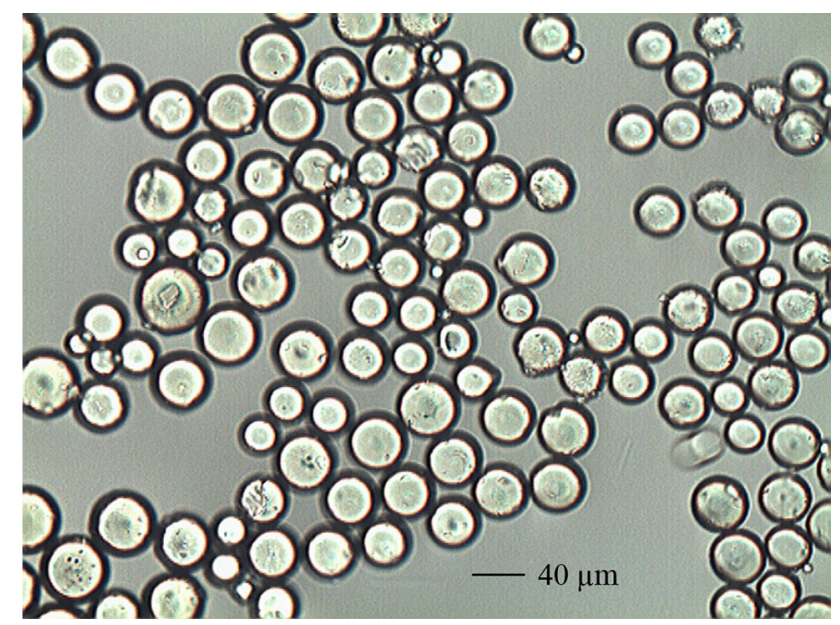

Fig. 2 Image of parent polymeric microspheres (BES.DM + GMA)

similar. Vibrations of $-\mathrm{OH}$ are visible as broad band in the range of $3600-3000 \mathrm{~cm}^{-1}$. Significant extension of this band (even up to $2500 \mathrm{~cm}^{-1}$ ) is the result of the presence of chelating and intramolecular hydrogen bonding. In the range of $1300-1000 \mathrm{~cm}^{-1}$ signals of $\mathrm{C}-\mathrm{O}$ stretching vibrations are observed. They are characteristic of functional groups of alcohols, ethers and esters, which are present in the structure of BES.DM molecule. All the

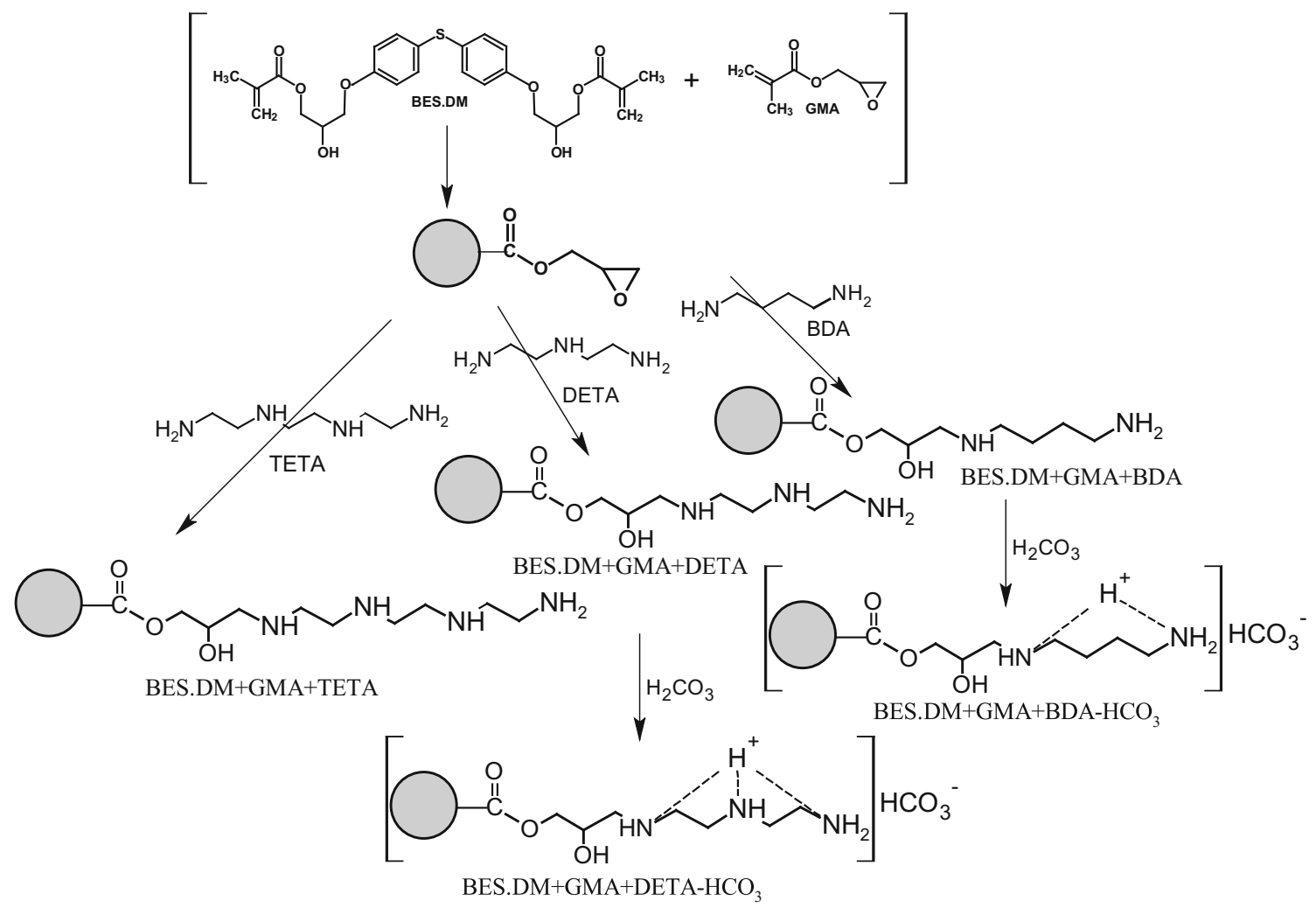

Fig. 1 Synthesis scheme of the studied copolymers 
Table 2 Porous structure parameters of the studied polymeric materials

\begin{tabular}{lllll}
\hline & $\mathrm{S}_{\mathrm{BET}}\left(\mathrm{m}^{2} / \mathrm{g}\right)$ & $\mathrm{V}_{\mathrm{t}}\left(\mathrm{cm}^{3} / \mathrm{g}\right)$ & $\mathrm{V}_{\mathrm{mi}}\left(\mathrm{cm}^{3} / \mathrm{g}\right)$ & $\mathrm{W}(\mathrm{nm})$ \\
\hline BES.DM + GMA & 39.1 & 0.215 & 0.001 & 20.8 \\
BES.DM + GMA + TETA & 27.0 & 0.220 & 0.001 & 28.9 \\
BES.DM + GMA + BDA + $\mathrm{HCO}_{3}{ }^{-}$ & 54.9 & 0.384 & 0.002 & 25.3 \\
BES.DM + GMA + DETA + $\mathrm{HCO}_{3}{ }^{-}$ & 52.7 & 0.352 & 0.002 & 24.3 \\
\hline
\end{tabular}

$V_{t}$ total pore volume

$V_{m i}$ micropore volume

$W$ average pore size
Fig. 3 Pore size distribution curves of the synthesized copolymers
Fig. 4 ATR spectra of the parent polymer and its derivatives
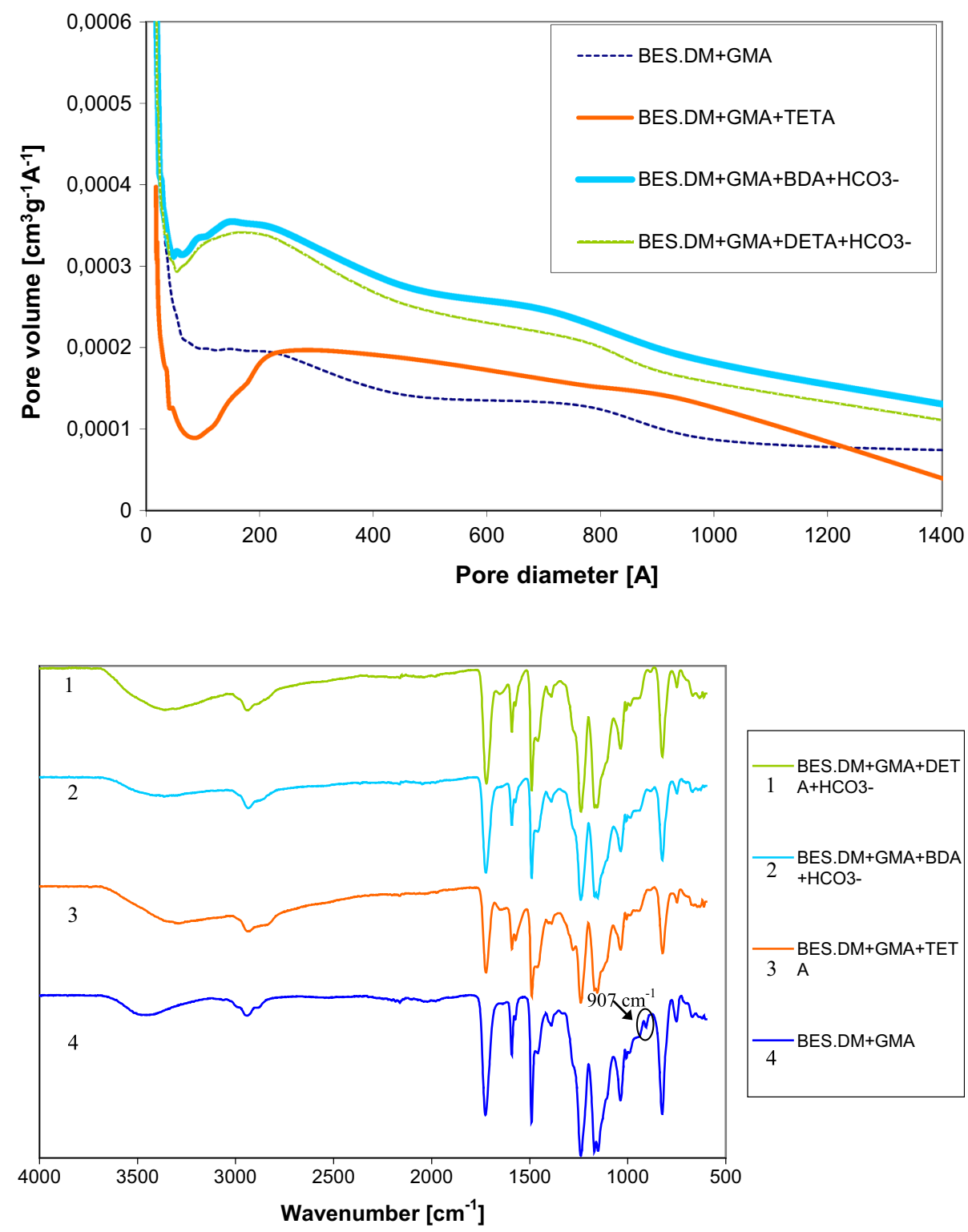

studied materials possess strong and narrow bands at about $1720 \mathrm{~cm}^{-1}$, which confirm the presence of $\mathrm{C}=\mathrm{O}$ bonds. Signals characteristic for aromatic rings are observed in the range of $1625-1430 \mathrm{~cm}^{-1}$ and at about $824 \mathrm{~cm}^{-1}$ (Socrates 2001).
After modification, some differences in the spectra can be observed. Parent polymer possesses epoxy rings which give their signals at $907 \mathrm{~cm}^{-1}$. In modification process, epoxy groups react with amines (BDA, DETA, TETA) and ring opening reaction takes place. For this reason, the band 
characteristic for epoxy groups is absent in the spectra of the modified polymers. After addition of the amines to the parent polymer, only little changes in the spectra of the modified materials are observed. Relative intensities of signals given by methyl and methylene groups (3000-2840 $\mathrm{cm}^{-1}$ ) are changed. A small band connected with amine $\mathrm{N}-\mathrm{H}$ deformation vibrations is observed at about $1650 \mathrm{~cm}^{-1}$. Weak signals of $\mathrm{N}-\mathrm{H}$ and $-\mathrm{NH}_{2}$ groups (at $3360 \mathrm{~cm}^{-1}$ ) appear on the broad band of $-\mathrm{OH}$ group (3600-3000 $\mathrm{cm}^{-1}$ ). This effect is better visible in the case of BES.DM + TETA, which possesses free primary amine groups. After addition of an $\mathrm{HCO}_{3}{ }^{-}$ion, the intensity of this band decreases as signals of secondary amine bonds are weaker. Most of the characteristic bands for bicarbonate ion (3300-2000; 1700-1600; 1420-1400; 1370-1290; and $660 \mathrm{~cm}^{-1}$ ) are situated in the same region as $\mathrm{C}-\mathrm{O}$, and $\mathrm{C}=\mathrm{O}$ signals of organic compounds (Socrates 2001). For this reason, the presence of these species cannot be confirmed by ATR-FITR spectra.

\subsubsection{Characteristic of surface chemistry}

For better characterization of surface chemical activity of the materials presented, elemental analysis, and measurements of $\mathrm{pH}$ of aqueous solution and ion exchange capacity were performed. The obtained results are collected in Table 3 .

The values of CEC and AEC for parent polymer are similar. Its ability to ion exchange is the effect of the presence of epoxy rings. Both in basic and acidic solution, the ring opening reaction takes place and, as a consequence, ion exchange capacity appears. The values of $\mathrm{pH}$ and AEC for modified materials strongly depend on nitrogen content introduced to the polymeric structure by amines. The basic character of amine is responsible for the $\mathrm{H}^{+}$bonding ability and the $\mathrm{pH}$ increase in water solution, therefore negative CEC is observed for the polymer BES.DM + GMA + TETA. For the same reason, CEC values for polymers modified with carbon dioxide are unexpectedly low. The $\mathrm{HCO}_{3}{ }^{-}$species can dissociate releasing $\mathrm{H}^{+}$ions, but the basic character of amine groups is stronger. Consequently, only little difference between
CEC values, but significant difference in $\mathrm{pH}$ for the polymers BES.DM + GMA + DETA $+\mathrm{HCO}_{3}{ }^{-}$and $\mathrm{BES} . \mathrm{DM}+\mathrm{GMA}+\mathrm{BDA}+\mathrm{HCO}_{3}{ }^{-}$is observed.

\subsubsection{TG/DTA analysis}

Thermal stabilities and degradation behaviours of copolymers were studied by means of TG/DTA analyses (Figs. 5, $6)$. It can be seen that the initial mass loss of copolymer ranges from $120{ }^{\circ} \mathrm{C}$ for BES.DM + GMA + TETA to about $220{ }^{\circ} \mathrm{C}$ for the other materials. The final decomposition temperature $\left(\mathrm{T}_{\mathrm{f}}\right)$ is in the range of $500-800{ }^{\circ} \mathrm{C}$.

The DTG curves contain two separated degradation steps. In case of the parent copolymer (BES.DM + GMA) and those modified with TETA, peaks of the first decomposition stage are observed in the maximum of mass loss $\left(\mathrm{T}_{1}\right)$ at 245 and $180{ }^{\circ} \mathrm{C}$, while the second decomposition stage $\left(\mathrm{T}_{2}\right)$ takes place at 410 and $400{ }^{\circ} \mathrm{C}$, respectively.

For copolymers modified with groups $-\mathrm{HCO}_{3}{ }^{-}$, the DTA curves have some different course. The first decomposition peak is observed in the maximum of mass loss $\left(\mathrm{T}_{1}\right)$ at 360 and $385^{\circ} \mathrm{C}$, while the second decomposition stage $\left(\mathrm{T}_{2}\right)$ takes place at 540 and $630{ }^{\circ} \mathrm{C}$.

The first stage of degradation $\left(\mathrm{T}_{1}\right)$ is associated with the releasing of volatile components from copolymers and decomposition of functional groups, whereas for the second one $\left(\mathrm{T}_{2}\right)$, the process of total degradation of polymeric network is responsible.

\subsection{The phenols recovery studies}

Results of the SPE experiments are presented in Fig. 7a-d. As was expected, the obtained data revealed the influence of the modification of the polymeric surface on the sorption properties.

The recovery curves obtained for parent polymer (Fig. 7a) show typical adsorption-desorption behaviour for phenolic compounds, which is a consequence of their different solubility in water and capability to dissociate. Some physical properties of the phenol and its chlorinated derivatives were described in previous work (Sobiesiak et al. 2006). Slightly soluble in water, trichlorophenol

Table 3 Characteristic of chemical structure of the polymeric materials

\begin{tabular}{|c|c|c|c|c|c|c|}
\hline & $\mathrm{C}(\%)$ & $\mathrm{H}(\%)$ & $\mathrm{N}(\%)$ & $\mathrm{pH}$ & $\mathrm{CEC}(\mathrm{mmol} / \mathrm{g})$ & $\mathrm{AEC}(\mathrm{mmol} / \mathrm{g})$ \\
\hline BES.DM + GMA & 59.74 & 6.44 & & 4.56 & 0.30 & 0.41 \\
\hline BES.DM + GMA + TETA & 53.57 & 7.45 & 8.13 & 9.91 & -1.91 & 3.54 \\
\hline $\mathrm{BES} \cdot \mathrm{DM}+\mathrm{GMA}+\mathrm{BDA}+\mathrm{HCO}_{3}^{-}$ & 58.31 & 7.09 & 3.06 & 5.81 & 0.21 & 2.04 \\
\hline BES.DM + GMA + DETA $+\mathrm{HCO}_{3}{ }^{-}$ & 55.50 & 7.16 & 4.21 & 8.83 & 0.15 & 2.48 \\
\hline
\end{tabular}

$C E C$ cation exchange capacity

$A E C$ anion exchange capacity 
Fig. 5 Thermogravimetric curves of the studied materials

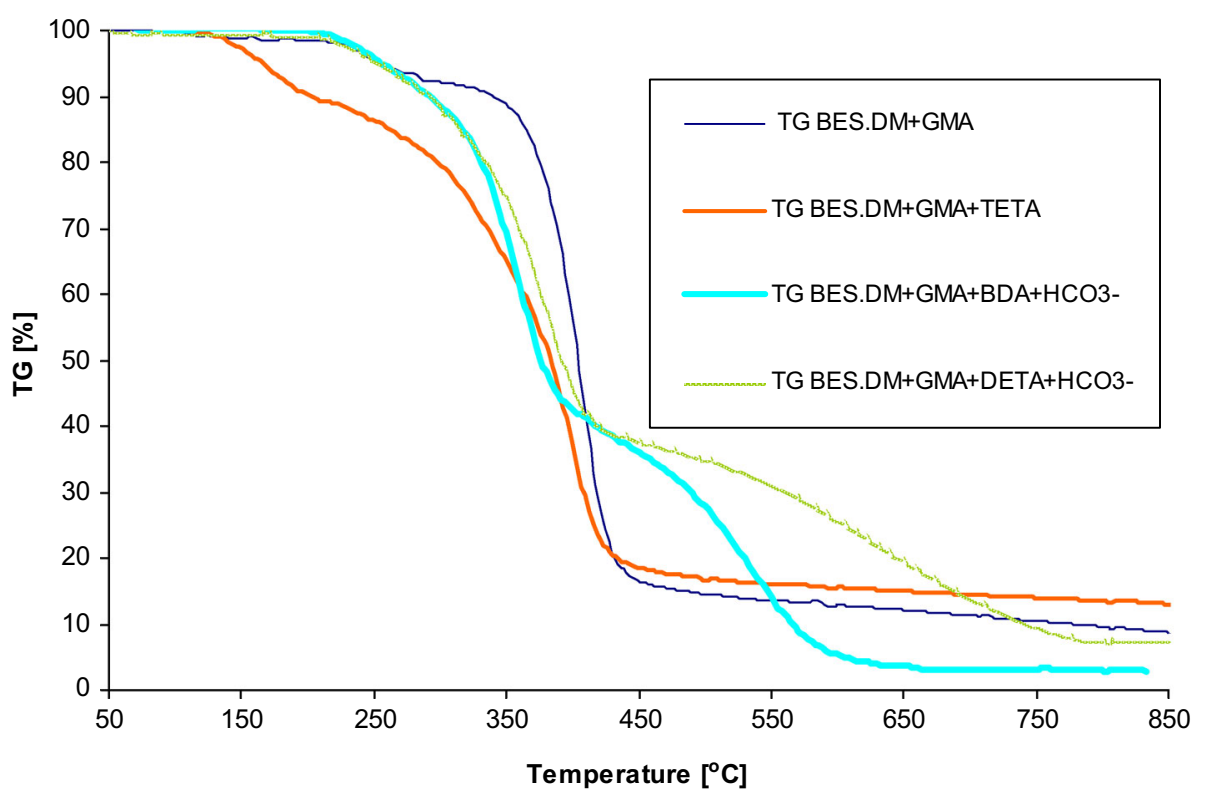

Fig. 6 DTG curves of the presented copolymers

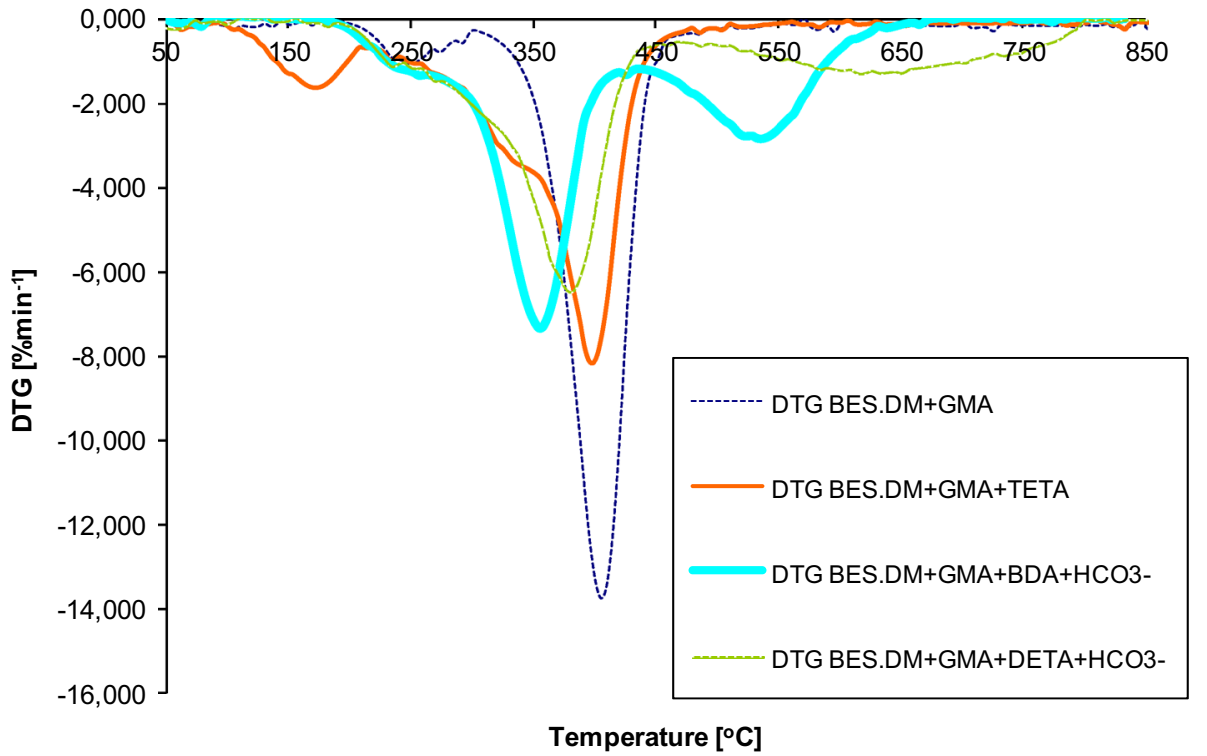

(TChP) possesses the best affinity to the polymeric surface. Sorption of phenol (PH), which dissolves in water much better than TChP, is very small. Along with increasing volume of sample solution, gradual decrease of recovery values for all the phenols is observed. Breakthrough for this sorbent occurs gradually in a wide range of sample volumes, from $200 \mathrm{~mL}$ (for $\mathrm{PH}$ and $\mathrm{ChP}$ ) to $800 \mathrm{~mL}$ (for TChP). For TChP the curve has a different course. Firstly, increase of recovery and next wide maximum can be observed. It means that some small amount of adsorbate is strongly bonded to the adsorbent and its elution is hindered.

The sorption capability drastically diminished after modification with TETA (Fig. 7b). Maximum recovery did not exceed $30 \%$, probably due to the reduction of specific surface area and the increase of large pores in the porous structure. Moreover, free pairs of electrons present in TETA molecules may cause repulsive forces between amine groups and $\pi$-electrons of aromatic rings from phenols.

Despite that, it is worth noticing that the affinity of TChP and DChP toward the polymer surface changed, especially in the range of small volumes of samples (up to $400 \mathrm{~mL}$ ). In this range, better sorption is observed for 2,4dichlorophenol. The recoveries of $\mathrm{DChP}$, and to a lower extent of $\mathrm{ChP}$ and $\mathrm{PH}$, decrease with the increasing volume of sample solution. In the case of TChP, an inverse 
Fig. 7 Recovery curves of the phenols obtained for: a the parent polymer; $\mathbf{b}$ the polymer modified with TETA; $\mathbf{c}$ the polymer modified with BDA and $\mathrm{CO}_{2} ; \mathbf{d}$ the polymer modified with DETA and $\mathrm{CO}_{2}$

\section{BES.DM+GMA}

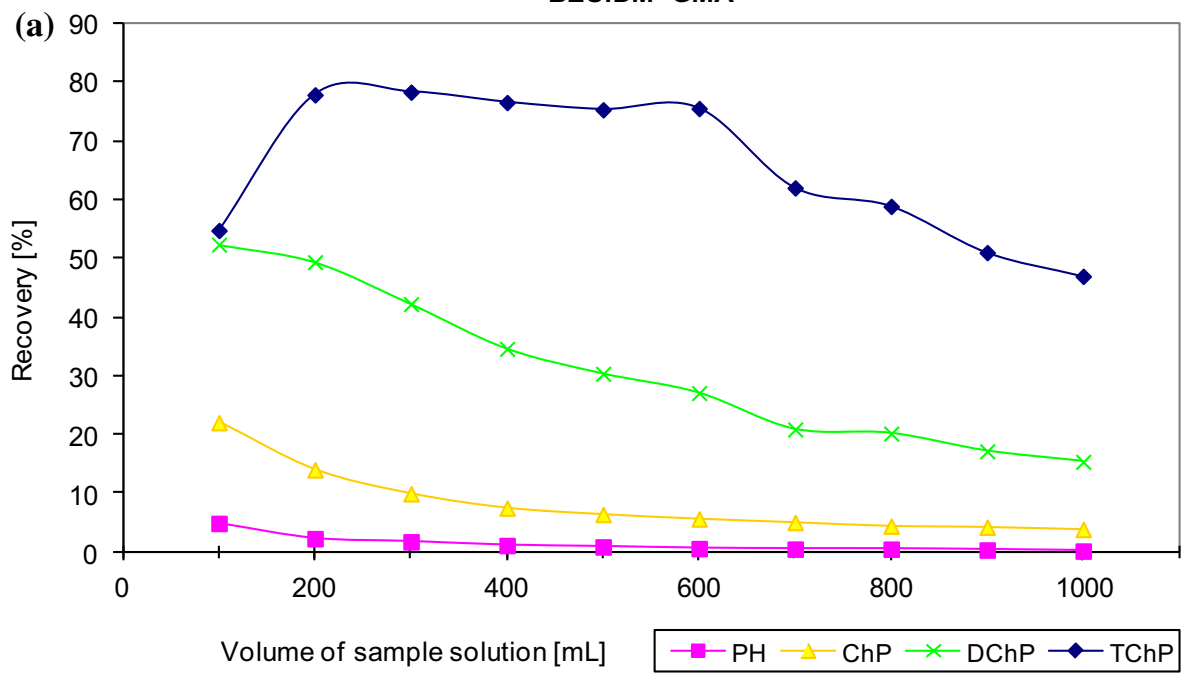

BES.DM+GMA+TETA

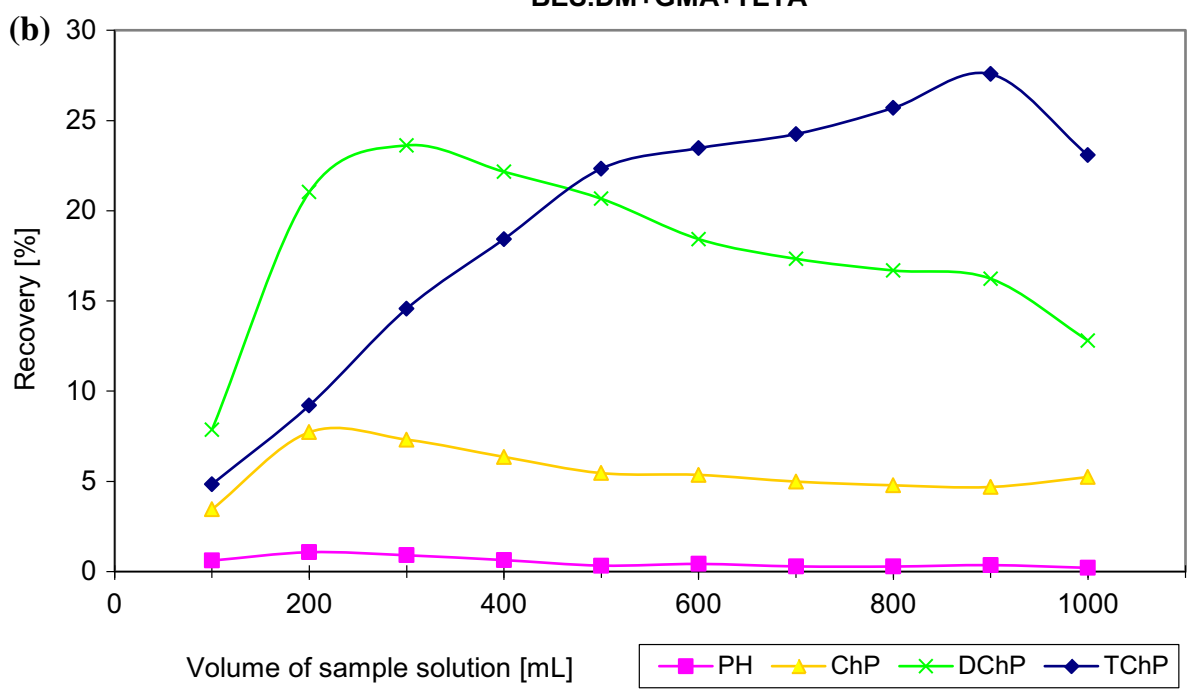

tendency is observed-the recoveries increase with increasing sample volume. This phenomenon proves competition for active sites on the sorbent surface between TChP and the rest of the phenolics molecules.

In the case of polymers modified with the amines (BDA and DETA) and the bicarbonate, competition between the molecules is even more intensive. Now, the best affinity to the surfaces of these materials is shown by 2,4dichlorophenol. Its recoveries are very high (85-100\%) in the wide range of sample solution volumes $(100-900 \mathrm{~mL})$. Maximum recovery of 2,4,6-trichlorophenol is reached at 800-900 mL for the BES.DM + GMA + BDA $+\mathrm{HCO}_{3}{ }^{-}$ polymer. For the BES.DM + GMA + DETA $+\mathrm{HCO}_{3}{ }^{-}$ polymer recovery is still on the increase at $1100 \mathrm{~mL}$. Moreover, these two sorbents present better sorption properties toward 2-chlorophenol in comparison to the parent polymer. Maximum recovery for ChP exceeds 60 and $55 \%$, respectively. Ascending part of recovery curves for $\mathrm{PH}, \mathrm{ChP}, \mathrm{DChP}$ is probably due to relatively short time of interactions of these compounds with the surface of the studied adsorbent. For TChP this effect can be explained by the phenomenon of competition for the active sites between testing compounds.

The observed differences in the chlorophenols' affinity towards the polymers studied are the result of changes in the chemical structure of the sorbents that took place during the modification steps. Both porous structure and surface functional groups have strong influence on process of sorption of phenolic compounds. That is observed as the changes in the course of the recovery curves. After addition of the amine, the polymeric surface became basic, which promoted dissociation of the testing compounds and in consequence a decrease of sorption. Introduction of $\mathrm{HCO}_{3}{ }^{-}$moieties improved sorption, because part of $-\mathrm{NH}_{2}$ 
Fig. 7 continued
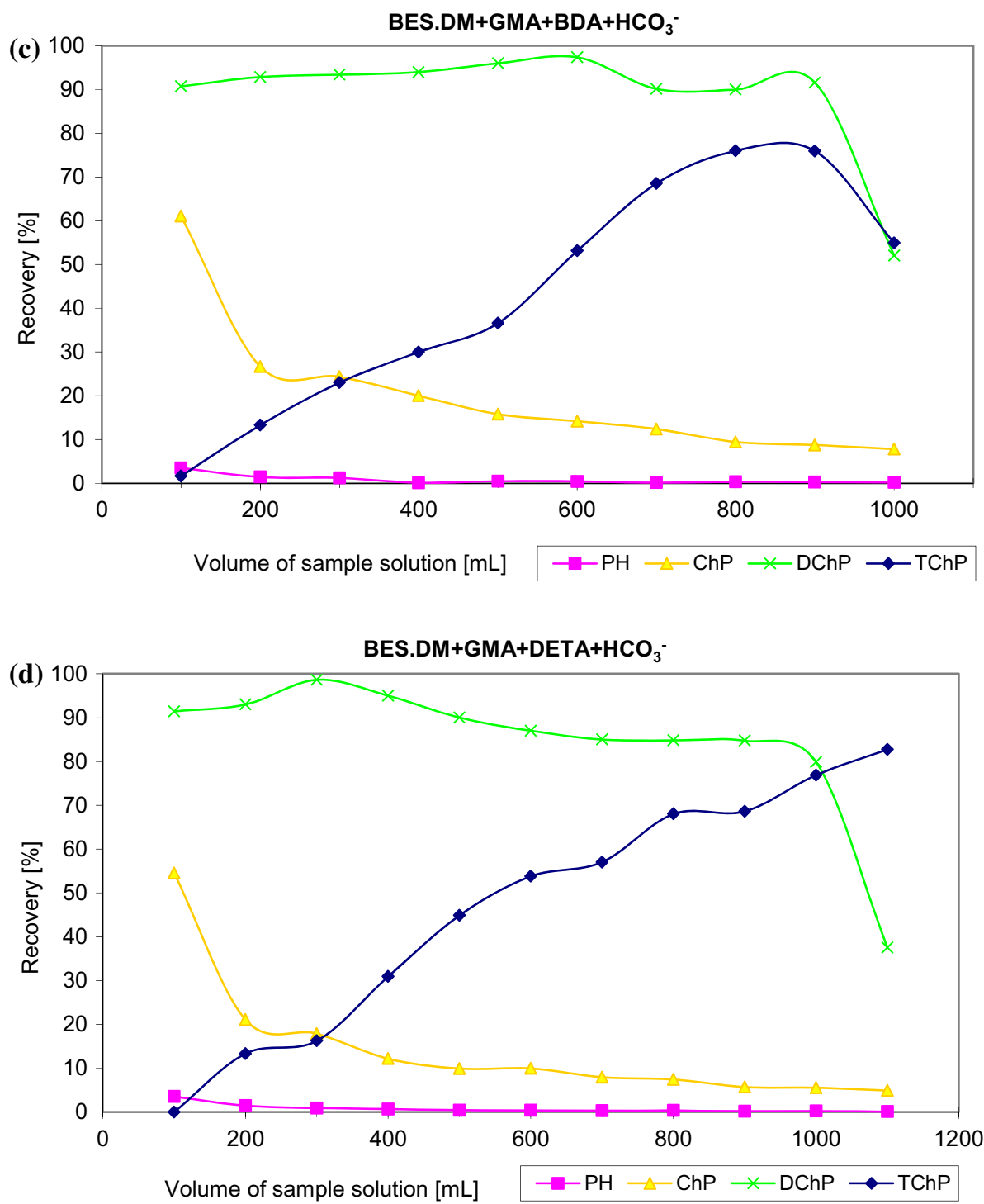

groups reacted and the polymeric surface became less basic. Moreover, bicarbonate groups are able to dissociate, causing shift the equilibrium dissociation reaction of chlorophenol toward unionized form.

Phenol seems to be insensitive to chemical modifications performed on the surface of BES.DM + GMA. Properties such as good solubility in water, high ability of dissociation and low value of $\log \mathrm{K}_{\mathrm{ow}}$ make sorption of this compound ineffective in the studied materials. Little amounts of phenol are adsorbed as a result of aromatic rings $\pi-\pi$ interactions.

\section{Conclusions}

This study presents the way of preparation and characterisation of three new polar sorbents with ability to adsorb chlorophenols. Advantage of these materials is that, they can be used repeatedly as the desorption and regeneration is possible.

New porous copolymeric microspheres were prepared by suspension-emulsion polymerization BES.DM with GMA. Multistage modification of the obtained adsorbent was made. Firstly, the copolymers were modified with amines (BDA, EDTA, TETA) in the epoxide ring opening reaction and next successful modification of primary amino groups by addition of $\mathrm{HCO}_{3}^{-}$was carried out. When $\mathrm{HCO}_{3}{ }^{-}$groups were introduced to the structures, the sorption capability increased and changes in affinity toward chlorophenols in comparison to the amine derivatives were additionally observed.

Both amines and bicarbonate groups were able to take part in the dissociation process and alter the $\mathrm{pH}$ of aqueous solution. As a consequence, the dissociation of chlorophenols was shifted to unionized forms that have positive influence on their sorption. This effect is especially 
visible in the case of the most toxic 2,4-dichlorophenol for which the best recovery results were achieved.

Open Access This article is distributed under the terms of the Creative Commons Attribution 4.0 International License (http://crea tivecommons.org/licenses/by/4.0/), which permits unrestricted use, distribution, and reproduction in any medium, provided you give appropriate credit to the original author(s) and the source, provide a link to the Creative Commons license, and indicate if changes were made.

\section{References}

An, F., Du, R., Wang, X., Wan, M., Dai, X., Gao, J.: Adsorption of phenolic compounds from aqueous solution using salicylic acid type adsorbent. J. Hazard. Mater. 201-2, 74-81 (2012)

Boehm, H.: Some aspects of the surface chemistry of carbon blacks and other carbons. Carbon 32, 759-769 (1994)

Castillejos, E., Rodríguez-Ramos, I., Sánchez, M.S., Muñoz, V., Guerrero-Ruiz, A.: Phenol adsorption from water solutions over microporous and mesoporous carbon surfaces: a real time kinetic study. Adsorption 17, 483-488 (2011)

Drinking Water Directive 80/778/EEC, Commission of the European Communities, Brussels (1980)

Feng, Q.-Z., Zhao, L.-X., Yan, W., Lin, J.-M., Zheng, Z.-X.: Molecularly imprinted solid-phase extraction combined with high performance liquid chromatography for analysis of phenolic compounds from environmental water samples. J. Hazard. Mater. 167, 282-288 (2009)

Fontanals, N., Marcé, R.M., Borrull, F.: New materials in sorptive extraction techniques for polar compounds. J. Chromatogr. A 1152, 14-31 (2007)

Gawdzik, B., Sobiesiak, M., Puziy, A.M., Poddubnaya, O.I.: Carbon sorbents derived from porous polymers for off-line preconcentration of chlorophenols from water. J. Liq. Chromatogr. Relat. Technol. 27, 1027-1041 (2005)

GESTIS Substance Database. Institut für Arbeitsschutz der Deutschen Gesetzlichen Unfallversicherung (IFA). http://gestis-en.itrust.de/ $\mathrm{nxt} /$ gateway.dll/gestis_en/010430.xml? $\mathrm{f}=$ templates $\$ \mathrm{fn}=$ default. $\mathrm{htm} \$ 3.0$

László, K., Podkościelny, P., Dąbrowski, A.: Heterogeneity of polymer-based active carbons in adsorption of aqueous solutions of phenol and 2,3,4-trichlorophenol. Langmuir 19, 5287-5294 (2003)

Lu, Q., Sorial, G.A.: Adsorption of phenolics on activated carbonimpact of pore size and molecular oxygen. Chemosphere $\mathbf{5 5}$, 671-679 (2004)

Lu, Q., Sorial, G.A.: The effect of functional groups on oligomerization of phenolics on activated carbon. J. Hazard. Mater. 148, 436-445 (2007)

Olaniran, A.O., Igbinosa, E.O.: Chlorophenols and other related derivatives of environmental concern: properties, distribution and microbial degradation processes. Chemosphere 83, 1297-1306 (2011)

Pan, B., Zhang, C.X., Zhang, W.M., Zheng, J.Z., Pan, B.J., Chen, J.L., Zhang, Q.X.: Adsorption of phenolic compounds from aqueous solution onto a macroporous polymer and its aminated derivative: isotherm analysis. J. Hazard. Mater. 121, 233-241 (2005)

Podkościelna, B.: Synthesis, modification, and porous properties of new glycidyl methacrylate copolymers. J. Appl. Polym. Sci. 120, 3020-3026 (2011)

Podkościelna, B.: The use of bis[4(2-hydroxy-3-methacryloyloxypropoxy)phenyl]sulfide in preparation of microspheres with pendant amine groups as a heavy metal sorbent. Sep. Sci. Technol. 48, 1699-1708 (2013)

Podkościelna, B., Bartnicki, A., Gawdzik, B.: Porous microspheres, copolymers of bis[4-(2-hydroxy-3-methacryloyloxypropoxy) phenyl]sulfide, and divinylbenzene as stationary phase for HPLC. J. Appl. Polym. Sci. 111, 1257-1267 (2009)

Podkościelny, P., Nieszporek, K.: Adsorption of phenols from aqueous solutions: Equilibria, calorimetry and kinetics of adsorption. J. Colloid Interf. Sci. 354, 282-291 (2011)

PubChem, Public Chemical Database, http://pubchem.ncbi.nlm.nih. gov/summary/summary.cgi?cid $=6914 \& l o c=e c \_r c s$

Puziy, A.M., Poddubnaya, O.I., Sobiesiak, M., Gawdzik, B.: Structural and surface heterogeneity of phosphorus-containing polyimide-derived carbons: effect of heat treatment temperature. Adsorption 19, 717-722 (2013)

Rodil, R., Quintana, J.B., Cela, R.: Oxidation of synthetic phenolic antioxidants during water chlorination. J. Hazard. Mater. 199-200, 73-81 (2012)

Rodríguez, I., Llompart, M.P., Cela, R.: Solid-phase extraction of phenols. J. Chromatogr. A 885, 291-304 (2000)

Simpson, N.J.K.: Solid-Phase Extraction-Principles. Techniques and Applications. Marcel Dekker. Inc., New York (2000)

Sobiesiak, M., Gawdzik, B., Puziy, A.M., Poddubnaya, O.I.: Polymerbased carbon adsorbents obtained from copolymer of 4,4' bis(maleimidodiphenyl)methane and divinylbenzene for use in SPE. Chromatographia 64, 175-181 (2006)

Sobiesiak, M., Podkoscielna, B.: Preparation and characterization of porous DVB copolymers and their applicability for adsorption (solid-phase extraction) of phenol compounds. Appl. Surf. Sci. 257, 1222-1227 (2010)

Sobiesiak, M.: Bead-shaped porous polymers containing bismaleimide - their physico-chemical characteristics and sorption properties towards chlorophenols. Polish J. Appl. Chem. 55, 25-32 (2011)

Sobiesiak, M.: Nanoporous carbons obtained by carbonization of copolymers impregnated by salts. Adsorption 19, 349-356 (2012)

Socrates, G.: Infrared and Raman characteristic group frequencies: tables and charts, 3rd edn. Wiley, Chichester (2001)

Zhong, W., Wang, D., Xu, X.: Phenol removal efficiencies of sewage treatment processes and ecological risks associated with phenols in effluents. J. Hazard. Mater. 217-218, 286-292 (2012) 